\title{
Problems in Publishing a Popular Journal in a Small Country
}

Pierre Gugnon and Jacques Sauval

Société Royale Belge d'Astronomie, Observatoire Royal de Belgique, Avenue circulaire, 3, B-1180 Brussels, Belgium

Every journal with a small circulation has the same problems and Ciel et Terre is no exception. The basic cause is the same everywhere: the first thousand copies is far more expensive than the second and subsequent thousands by a factor of 6 to 8 times. This obviously arises from the cost of origination of material. All low-circulations journals have the same financial problems.

We have found that it is beneficial to periodically review the following points: the printing process, the format, the frequency, the editorial team, distribution, promotion, subscription fee, etc. In recent years we have found this process to lead to considerable savings.

Photolitho printing in A4 size has considerable advantages, and bi-monthly publication balances costs of postage against size of journal and reader satisfaction. Our journal is distributed to all subscribers and special numbers are sold by specialist booksellers and other astronomical groups, and sent free of charge to daily and weekly newspapers with a request that it be mentioned (with very good results).

Supplementary sources of income arise from special issues, such as annual ephemerides, and publications on the Sun, comets, etc. which can interest a wide public if they are properly promoted and if the selling cost is fixed low. This can be achieved because they are normal bi-monthly issues, so the additional numbers can be printed at relatively low costs. Advertisements are a useful but moderate source of income.

Other economies can be made by setting the text direct from disk, providing diagrams to final size, etc.

We would suggest that there could well be assistance between the various journals, perhaps even with exchange of material. If the editors of any astronomical journals wish to discuss any of the ideas that we raise, we shall be pleased to help. 